\title{
Prokaryotic expression, purification and characterization of human cyclooxygenase-2
}

\author{
XIANGZHI LIAO ${ }^{1}$, WENHAN WANG ${ }^{1}$, CHUANXI FAN $^{1}$, NING YANG $^{1}$, JIALIANG ZHAO $^{1}$, \\ YING ZHANG $^{1,2}$, RUIJUAN GAO ${ }^{1}$, GUANNAN SHEN $^{1}$, SIMIN XIA $^{1}$ and GUIYING LI ${ }^{1}$ \\ ${ }^{1}$ Key Laboratory for Molecular Enzymology and Engineering of the Ministry of Education, \\ College of Life Sciences, Jilin University, Changchun, Jilin 130012; ${ }^{2}$ Department of Pediatrics, \\ The First Hospital of Jilin University, Changchun, Jilin 130021, P.R. China
}

Received September 14, 2016; Accepted May 26, 2017

DOI: $10.3892 /$ ijmm.2017.3007

\begin{abstract}
Cyclooxygenase-2 (COX-2) is a key enzyme which catalyzes the conversion of arachidonic acid (AA) into prostaglandins (PGs). It plays an important role in pathophysiological processes, such as tumorigenesis, angiogenesis, inflammation and tumor cell drug resistance. Therefore, COX-2 has been viewed as an important target for cancer therapy. The preparation of COX-2 protein is an important initial step for the subsequent development of COX-2 inhibitors. In this study, we report a strategy to heterologously express truncated human COX-2 (trCOX-2) in Escherichia coli (E. coli) BL21(DE3) host cells. Following denaturation, purification and renaturation, we successfully obtained enzymatically active trCOX-2 containing 257 residues of the C-terminus. Homology modeling and molecular docking analyses revealed that trCOX-2 retained the predicted 3D catalytic domain structure and AA could still bind to its hydrophobic groove. Western blot analysis and ELISA indicated that the trCOX-2 still retained its characteristic antigenicity and binding activity, while COX assays revealed that trCOX-2 maintained its enzyme activity. On the whole, in this study, we provided a novel method to isolate trCOX-2 possessing AA binding and catalytic activities. This study thus lays a foundation to facilitate further investigations of COX-2 and offers a valuable method with which to achieve the prokaryotic expression of a eukaryotic membrane protein.
\end{abstract}

Correspondence to: Professor Guiying Li, Key Laboratory for Molecular Enzymology and Engineering of the Ministry of Education, College of Life Sciences, Jilin University, 2699 Qianjin Street, Changchun, Jilin 130012, P.R. China

E-mail: ligy@jlu.edu.cn

Abbreviations: COXs, cyclooxygenases; PGs, prostaglandins; AA, arachidonic acid; $\mathrm{PGE}_{2}$, prostaglandin $\mathrm{E}_{2}$; trCOX-2, truncated human COX-2

Key words: human cyclooxygenase, prokaryotic expression, inclusion bodies, purification, computer simulation

\section{Introduction}

The cyclooxygenases (COXs), also known as prostaglandin endoperoxide $\mathrm{H}$ synthases (PGHSs), are 67-72 kDa integral membrane proteins located on the endoplasmic reticulum (ER) and the nuclear envelope. COXs are fatty acid oxygenases and members of the myeloperoxidase superfamily (1-5). COXs are bifunctional enzymes and sequence homodimers; each monomer has COX (or bis-dioxygenase) activity and peroxidase (POX) activity via physically distinct COX and POX active sites $(1,3,5)$. COXs catalyze the conversion of arachidonic acid (AA) to $\mathrm{PGH}_{2}$, which is the initial rate-limiting step in prostaglandin (PG) biosynthesis (1-6). The production of $\mathrm{PGH}_{2}$ is a two-step reaction: AA binds inside the COX tunnel and reacts to form the intermediate $\mathrm{PGG}_{2}$ and $\mathrm{PGG}_{2}$ is bound and modified within the peroxidase active site to form the final product, $\mathrm{PGH}_{2}$ (3-7).

All vertebrates investigated to date possess two COX isoforms, COX-1 and COX-2. In most cases, COX-1 is expressed constitutively to produce PGs that mediate 'housekeeping' functions, whereas the expression of COX-2 is highly inducible in response to growth factors, tumor promoters or cytokines $(6,8)$. COX-2-derived PGs participate in a number of pathophysiological responses, such as inflammation, carcinogenesis and modulation of cell growth and survival (9). Increasing evidence has indicated that the induced expression and activation of COX-2 are observed in many types of tumor cells, and are involved in tumor progression and aggressiveness $(10,11)$. In addition, the expression of COX-2 was observed to be induced in cancer cells during anticancer chemoradiotherapies, resulting in drug resistance (11-13). Thus, the inhibition of COX-2 may provide a very significant therapy which may benefit a large proportion of the patient population (10). While broad spectrum COX-2-inhibiting non-steroidal antiinflammatory drugs (NSAIDs), and COX-2-specific inhibitors have been successfully established $(11,12)$, both of these are known to cause side-effects, such as myocardial infarction (11). Therefore, there still remains an urgent need to develop anti-COX-2 therapies with reduced or no side-effects.

The preparation of COX-2 protein is the initial step for the development of COX-2 inhibitors. A eukaryotic heme-containing and membrane-bound protein, COX-2 is expressed at a rather 
low level in native hosts. Heterologous expression is the only efficient strategy with which to obtain a large amount of human COX-2 protein. Generally, the most frequently used heterologous expression systems include prokaryotic, yeast, plant-based, insect/baculovirus and mammalian expression systems, as well as expression in eukaryotic organisms $(14,15)$. With the progression of COX-2 structural studies (3-6), the insect/baculovirus expression system has become the most widespread method for acquiring high quality functional products (16-19). However, several limitations of the insect/baculovirus system, including a relatively high cost, methodological challenges and relatively low yields obtained using this system, limit its use for largescale fermentation and more widespread application. Moreover, protein synthesis rates are generally much faster in prokaryotes than in eukaryotes (20). Thus, bacterial hosts are preferred, due to their rapid growth rate, their capacity for continuous fermentation, high-level post-induction target protein expression and a relatively low cost (14,20-26). However, to date, and at least to the best of our knowledge, limited research has been carried out to characterize and purify human COX-2 expressed in prokaryotic cells (27).

In this study, a truncated form of human COX-2, containing 257 residues of the C-terminus was cloned, and it exhibited high-level heterologous expression in Escherichia coli (E. coli) BL21(DE3) cells using the pET28b(+) expression vector system. In addition, the antigenicity and the COX activity of truncated human COX-2 (trCOX-2) products were validated and these results demonstrate the reliability of this method to obtain functional COX-2 products from a prokaryotic expression system.

\section{Materials and methods}

Materials. BamHI, HindIII and T4 DNA ligase were all purchased from Takara Biotechnology Co., Ltd. (Dalian, China). A Ni ${ }^{2+}$-NTA Superflow Cartridge was purchased from Qiagen (Valencia, CA, USA). PD-10 desalting columns were obtained from Amersham Pharmacia Biotech, Inc. (Piscataway, NJ, USA). Coomassie Brilliant Blue R-250 was obtained from Sigma-Aldrich; Merck KGaA (Darmstadt, Germany). Anti-COX-1 (sc-166573) and anti-COX-2 antibodies (sc-166475) were both purchased from Santa Cruz Biotechnology, Inc. (Santa Cruz, CA, USA). Mouse anti-His monoclonal antibody (M0812-3) was purchased from Hangzhou HuaAn Biotechnology Co., Ltd. (Hangzhou, China). Horseradish peroxidase (HRP)-conjugated anti-mouse immunoglobulin G (IgG; SA00001-1) was purchased from Proteintech (Chicago, IL, USA). AA was purchased from Alfa Aesar (Ward Hill, MA, USA). All other chemicals and reagents used were of highest purity.

Homology modeling and molecular docking. The three dimensional structure of trCOX-2 was modeled through homology modeling using a published murine COX-2 structure (PDB ID: 4RRW) as the template (28). The homology modeling and calibration of models was conducted online using the SWISS-MODEL server (29-32). The molecular docking was conducted online using the SwissDock server $(33,34)$ with AA (PubChem CID: 444899) designated as the ligand and the trCOX-2 modeled structure designated as the receptor. All structure files were visualized using PyMOL (installed on an Ubuntu
Linux system provided by Canonical Ltd.). Protein-ligand interactions were analyzed with the help of the PyMOL viewer.

Construction of pET28b-trCOX-2. The $771 \mathrm{bp}$ stretch of sequence at the 3 '-end of full-length human COX-2 gene was amplified to obtain $\operatorname{trCOX}-2$ using primers designed using Primer Premier 5.0 with the following sequences: forward, 5'-TAACGTGGATCCGGACCCAGAACTACTTT-3' and reverse, 5'-GACCCCAAGCTTATACAGTTCAGT-3'. The DNA fragment coding for trCOX-2 was cloned into the pET28b(+) vector (Novagen, Madison, WI, USA), containing 6 histidines at both the amino terminus and the C-terminus. The recombinant plasmid, pET28b-trCOX-2, was produced in the E. coli strain JM109 and sequenced by Sangon Biotech Co., Ltd. (Shanghai, China). This plasmid expresses a 305 amino acid stretch of trCOX-2, which contains 257 amino acids of the $\mathrm{C}$-terminus residue of human COX-2 and additional histidine tags.

Expression of trCOX-2 in E. coli strain BL21(DE3). The pET28b-trCOX-2 plasmid was transformed into $E$. coli BL21(DE3) cells and induced to express trCOX-2 according to our previous studies $(25,26)$. Briefly, E. coli BL21(DE3) cells were transformed with pET28b-trCOX-2 to obtain $E$. coli trCOX-2/BL21(DE3) that could express trCOX-2. E. coli trCOX-2/BL21(DE3) were grown in Luria-Bertani (LB) medium with $30 \mu \mathrm{g} / \mathrm{ml}$ kanamycin at $37^{\circ} \mathrm{C}$ with shaking until the optical density at $600 \mathrm{~nm}$ reached 0.6 . The cells were then stimulated with $1 \mathrm{mM}$ isopropyl $\beta$-D-1-thiogalactopyranoside (IPTG) for $2,3,4,6$ and $8 \mathrm{~h}$ at $30^{\circ} \mathrm{C}$ with shaking. E. coli trCOX-2/BL21(DE3) cells were harvested by centrifugation at $8,000 \mathrm{rpm}$ for $15 \mathrm{~min}$ at $4^{\circ} \mathrm{C}$ and lysed by sonication in buffer $\mathrm{A}$ containing $20 \mathrm{mM}$ sodium phosphate, $\mathrm{pH} 7.4,500 \mathrm{mM}$ $\mathrm{NaCl}, 10 \mathrm{mM}$ imidazole, $0.1 \mathrm{mM}$ phenylmethylsulfonyl fluoride (PMSF) and $1 \mathrm{mM} \beta$-mercaptoethanol. The lysates were fractionated by centrifugation at $15,000 \mathrm{rpm}$ for $15 \mathrm{~min}$ at $4^{\circ} \mathrm{C}$. The supernatant and precipitate were separately analyzed by $12 \%$ sodium dodecyl sulfate-polyacrylamide gel electrophoresis (SDS-PAGE) and stained with Coomassie Brilliant Blue R-250 to visualize the expression of trCOX-2. The average gray value of each band was detected and quantified using BandScan 5.0 software (Glyko Inc., Novato, CA, USA), and the results were expressed as the ratio of trCOX-2 to total proteins.

Denaturation of inclusion bodies. Inclusion bodies were washed sequentially with buffer B $(0.5 \%$ Triton X-100, $500 \mathrm{mM} \mathrm{NaCl}, 20 \mathrm{mM}$ sodium phosphate, $\mathrm{pH}$ 7.4) and buffer $\mathrm{C}$ (2 M urea, $500 \mathrm{mM} \mathrm{NaCl}, 20 \mathrm{mM}$ sodium phosphate, $\mathrm{pH}$ 7.4). The washed inclusion bodies were subsequently denatured in binding buffer $\mathrm{D}$ ( $8 \mathrm{M}$ urea, $20 \mathrm{mM}$ sodium phosphate, $\mathrm{pH} 7.4$, $500 \mathrm{mM} \mathrm{NaCl}, 0.1 \mathrm{mM}$ PMSF, $1 \mathrm{mM} \beta$-mercaptoethanol and $10 \mathrm{mM}$ imidazole) overnight at $4^{\circ} \mathrm{C}$. The soluble denatured inclusion body proteins were carefully collected by centrifugation at $15,000 \mathrm{rpm}$ at $4^{\circ} \mathrm{C}$ for $20 \mathrm{~min}$.

Purification and renaturation of inclusion body proteins. The soluble inclusion body proteins were applied to a $\mathrm{Ni}^{2+}-\mathrm{NTA}$ Superflow Cartridge (Qiagen) equilibrated with binding buffer. The column was next washed sequentially with binding buffer $\mathrm{D}$ followed by washing buffer ( $8 \mathrm{M}$ urea, $20 \mathrm{mM}$ sodium 
phosphate, pH 7.4, $500 \mathrm{mM} \mathrm{NaCl}, 0.1 \mathrm{mM}$ PMSF, $1 \mathrm{mM}$ $\beta$-mercaptoethanol and $40 \mathrm{mM}$ imidazole) and then eluted with elution buffer ( $8 \mathrm{M}$ urea, $20 \mathrm{mM}$ sodium phosphate, $\mathrm{pH} 7.4$, $500 \mathrm{mM} \mathrm{NaCl}, 0.1 \mathrm{mM}$ PMSF, $1 \mathrm{mM} \beta$-mercaptoethanol and $500 \mathrm{mM}$ imidazole). The purification of denatured trCOX-2 was monitored by analyzing aliquots of the collected samples using 12\% SDS-PAGE and then stained with Coomassie Brilliant Blue R-250. The desired eluted proteins were refolded as previously described (26). Briefly, the purified denatured trCOX-2 products were diluted 1:10 in refolding buffer $\mathrm{E}$ (42 mM Tris-HCl, pH 8.0, 62 mM HEPES, 2.5 mM DTT, $0.1 \mathrm{mM} \mathrm{CaCl}_{2}, 0.5 \mathrm{M}$ arginine) and slowly stirred on ice for $4 \mathrm{~h}$ to allow COX-2 renaturation to occur. The renatured trCOX-2 was stored at $-80^{\circ} \mathrm{C}$ following determination of protein concentration using the Bradford assay.

Western blot analysis. The samples were subjected to SDS-PAGE followed by electrophoretic transfer onto polyvinylidene difluoride (PVDF) membranes. Non-specific binding was blocked with blocking buffer containing PBST $[0.05 \%$ Tween-20 in phosphate-buffered saline (PBS)] with 5\% non-fat milk for $1 \mathrm{~h}$ at room temperature. The membranes were then incubated overnight at $4^{\circ} \mathrm{C}$ with antibodies specific either for the His-tag or COX-2 in PBST containing 5\% non-fat milk at the dilutions specified by the manufacturers. After washing 3 times with PBST, the membranes were incubated with HRP-conjugated secondary antibodies at a dilution of 1:5,000 in PBST containing 5\% non-fat milk for $1 \mathrm{~h}$ at room temperature. The membranes were subsequently washed 3 times with PBST and the protein bands were detected using a western blot detection system.

Enzyme-linked immunosorbent assay (ELISA). For ELISA, the purified trCOX-2 $(1-10 \mu \mathrm{g} / \mathrm{ml})$ was coated onto the surface of wells of a 96-well ELISA plate overnight at $4{ }^{\circ} \mathrm{C}$. The wells were then blocked with PBST containing $3 \%$ non-fat milk for $1 \mathrm{~h}$ at $37^{\circ} \mathrm{C}$. Following sequential incubation with a primary antibody (antibodies against COX-1 or COX-2) and HRP-conjugated IgG, the reaction was developed by the addition of o-phenylenediamine (OPD) and monitored using a microplate reader (Thermo Labsystems, Waltham, MA, USA) at a wavelength of $492 \mathrm{~nm}$. Wells coated with the same amount of BSA instead of trCOX-2 served as the negative control (NC).

COX assay. For measurement of COX activity, $\mathrm{O}_{2}$ consumed in the assay mixture was monitored using a dissolved oxygen detector (OXY5401S; Puyang, China) at $37^{\circ} \mathrm{C}(5,6)$. The standard assay mixture contained $6 \mathrm{ml}$ of $100 \mathrm{mM}$ Tris, $\mathrm{pH} 7.4,2 \mathrm{mM}$ phenol, $10 \mu \mathrm{M}$ hematin and $100 \mu \mathrm{M}$ of AA substrate. Reactions were initiated by the addition of up to $50 \mu \mathrm{g}$ of protein. The variation of the $\mathrm{O}_{2}$ concentration between the initiation and completion of the reaction was monitored and compared with the assay mixture without the enzyme sample. All of the above reactions were monitored in an anaerobic workstation.

\section{Results}

Design strategy for the expression of human $\mathrm{COX}-2$ at a high level in E. coli. Full-length human COX-2 contains 604 amino acids, beginning with the signal peptide, followed by an epidermal growth factor (EGF)-like domain, a membrane binding domain, a dimerization domain and the catalytic domain at the carboxyl terminus (1). Our preliminary data showed that it was very difficult to express full-length human COX-2 in E. coli after our group made several failed attempts to purify the full-length human COX-2 (data not shown). We surmised that these difficulties were due to the known phenomenon of heterogeneous membrane polarization observed in membrane proteins, as well as to the large size of the COX-2 target protein. Based on these factors, subsequent attempts were made to remove the non-catalytic domain using published knowledge of protein structures and function (34-38). As previous results have shown that the deletion of the N-terminal signal peptide could significantly increase protein expression levels in E. coli (19), to obtain a high yield of functional human COX-2 in E. coli, we designed a strategy to prepare a trCOX-2 possessing catalytic activity. Following the deletion of the $\mathrm{N}$-terminal 347 amino acid residues from full-length human COX-2, the remainder of the trCOX-2 should still possess the core catalytic portion of full-length $\mathrm{COX}-2$, including all important binding and catalytic sites (6).

\section{Computer simulation of trCOX-2}

Homology modeling and structure alignment of trCOX-2. To verify our design strategy of human trCOX-2, we conducted homology modeling of trCOX-2 with partial human COX-2 catalytic domain containing 257 residues of the C-terminus. Human COX-2 and murine COX-2 share $85 \%$ sequence identity and share highly conserved crystal structures. The trCOX-2 structure with 305 amino acids (containing some amino acids from the vector) was depicted according to SWISSMODEL using a database of the PDB entry 4RRW, as shown in Fig. 1A. The newly published crystal structure of murine COX-2 (PDB ID: 4RRW) was selected as the template, with a sequence similarity of $94 \%$ and a coverage of $79 \%$. It contains 242 amino acids aligning to the Arg-363 to Leu-604 stretch of trCOX-2. The modeled structure had a reasonable QMEAN4 score $(|\mathrm{QMEAN} 4|<1)$. The trCOX-2 is a monomer, while $4 \mathrm{RRW}$ is a homotetramer. The alignment between the trCOX-2 and one monomer of 4RRW, which are oriented in the same direction with homologous residue positions aligned are shown in Fig. 1B. According to the modeling structure, $3 \alpha$-helices in trCOX- 2 form the catalytic pocket, while $5 \alpha$-helices form the catalytic pocket in murine COX-2. Important residues, including Phe-381, Tyr-385, Trp-387, Val-523, Glu-524, Ser-530 and Leu-531, possess almost the same relative spatial relationships in both trCOX-2 and the template. Although the larger pocket of trCOX-2 may lead to weaker interactions and impaired enzyme activity, the remaining helices, especially the key residues, mainly adopt the same conformation compared with the template. These results indicate that the major catalytic domain is conserved in trCOX-2.

Docking of $A A$ to $\operatorname{trCOX}-2$. We then conducted molecular docking between AA and trCOX-2. The docking results (Fig. 2A) revealed that AA bound in the COX channel of trCOX-2, further elucidating the important catalytic residues of trCOX-2 which may exhibit enzyme activity. As there is no significant structural differences between the 

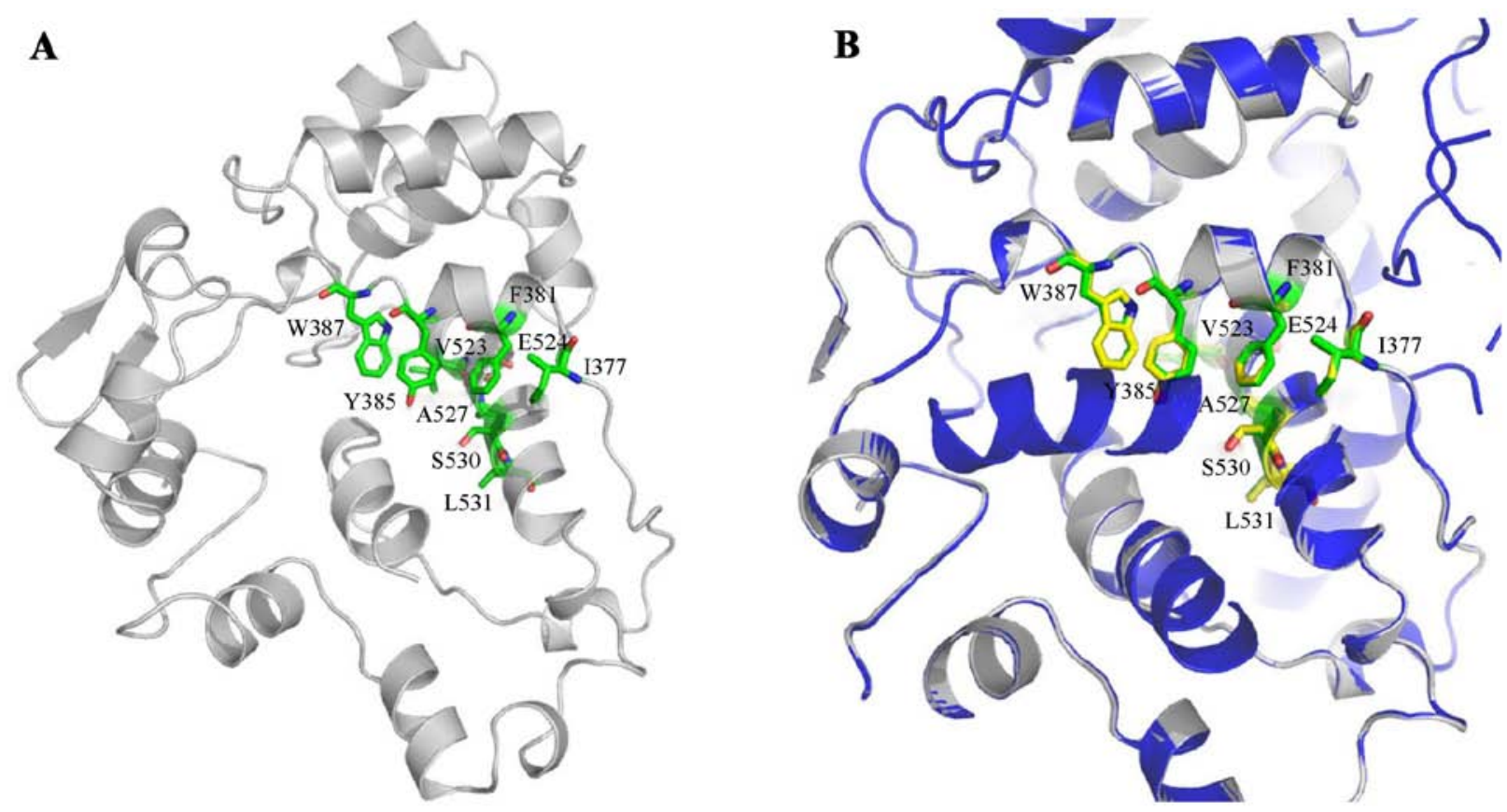

Figure 1. Homology modeling and structure alignment of truncated human cyclooxygenase-2 (trCOX-2). (A) The three-dimensional structure of trCOX-2 (gray). The key residues (green): Ile-377, Phe-381, Tyr-385, Trp-387, Val-523, Glu-524, Ala-527, Ser-530 and Leu-531. (B) The alignment of trCOX-2 and its template (PDB ID: 4RRW, blue) are amplified to show the core catalytic domain; corresponding key residues of 4RRW are shown in yellow. Generally, the conformations of these residues are superimposed. The structures were visualized using PyMOL version 1.6.x for Ubuntu.
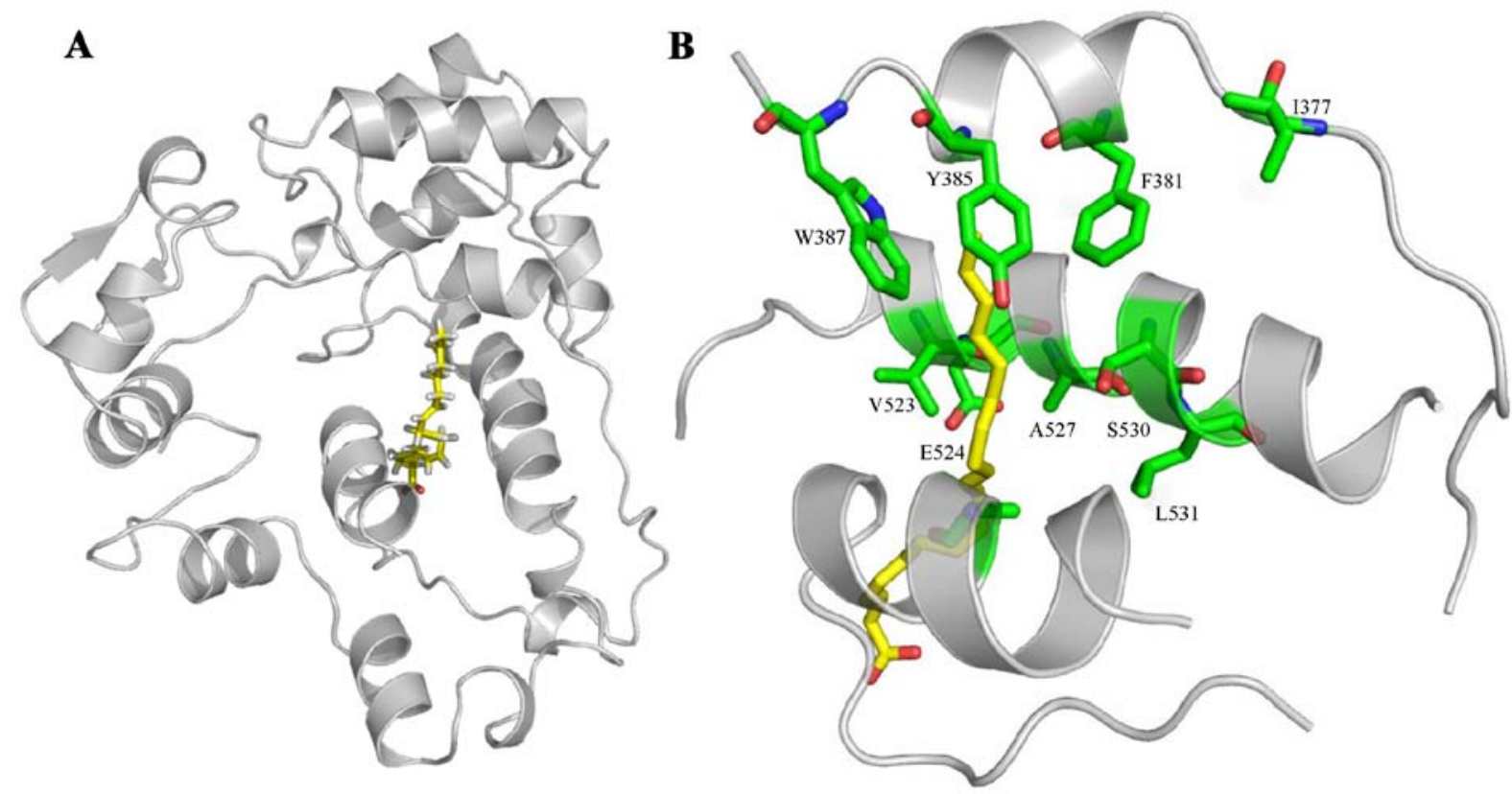

Figure 2. Molecular docking arachidonic acid (AA) to truncated human cyclooxygenase-2 (trCOX-2). (A) Overview of AA (yellow) bound to COX sites of trCOX-2 (gray). (B) The binding pocket (COX site) and the hydrophobic groove of trCOX-2 with AA. The key residues: Ile-377, Phe-381, Tyr-385, Trp-387, Val-523, Glu-524, Ala-527, Ser-530 and Leu-531 are shown in green.

core-binding pockets of muCOX-2 and trCOX-2, their similar binding structures raise the possibility that trCOX-2 retains enzyme activity $(4,6)$. As depicted in Fig. 2B, AA is oriented with its carboxylate moiety proximal to the COX-2 channel opening. Specifically, the AA $\omega$-end is located within the hydrophobic groove proximal to the Tyr-385 and Ser-530 residues positioned at the channel apex. Polar interactions are indicated between Tyr-385 and AA, Glu-524 and AA. Taken together, these results indicate that the hydrophobic groove and polar groups interact together to stabilize AA when it is bound within the COX channel.

Recombinant pET28b-trCOX-2 expression plasmid was constructed successfully. To prepare functional trCOX-2 


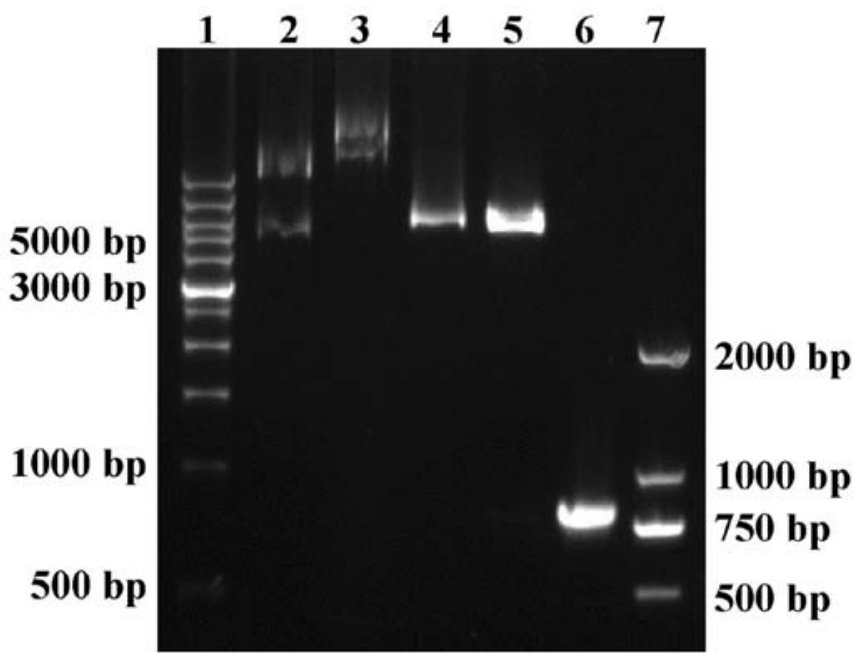

Figure 3. Construction of recombinant pET28b-truncated human cyclooxygenase-2 (trCOX-2) plasmid. Lane 1, wide range DNA marker; lane 2, pET28b plasmid; lane 3, pET28b-trCOX-2 plasmid; lane 4, pET28b plasmid digested with BamHI and HindIII; lane 5, pET28b-trCOX-2 plasmid digested with BamHI and HindIII; lane 6, trCOX-2 PCR products; and lane 7, BS2000 DNA marker.

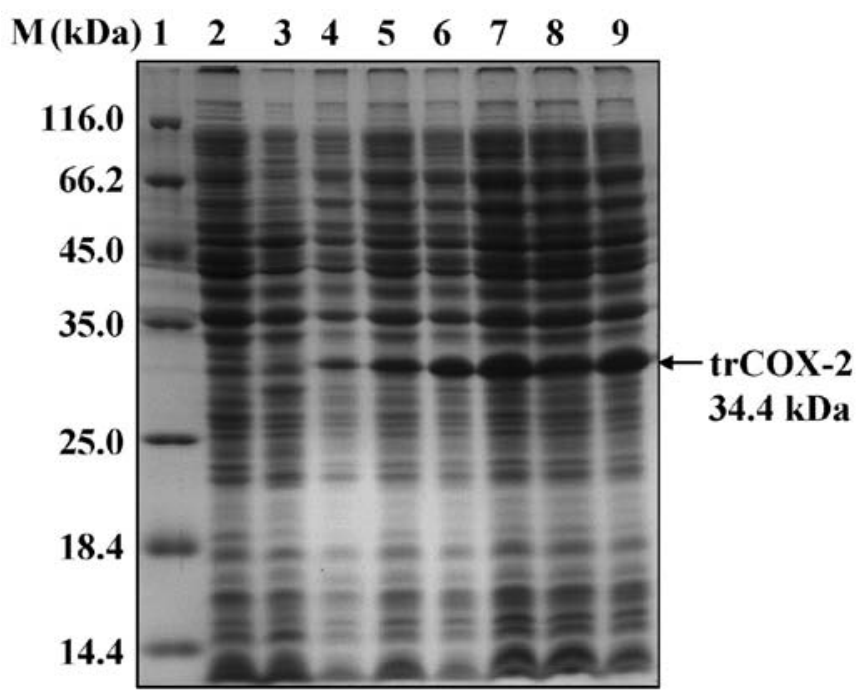

Figure 4. Analysis of truncated human cyclooxygenase-2 (trCOX-2) expression in Escherichia coli (E. coli) BL21(DE3) by 12\% SDS-PAGE. Lane 1, protein molecular weight standard; lane 2, cell lysate of $E$. coli BL21(DE3); lane 3, cell lysate of pET28b/BL21(DE3); lane4, celllysate of pET28b-trCOX-2/BL21(DE3) without induction; lanes 5-9, cell lysate of pET28b-trCOX-2/BL21(DE3) induced by isopropyl $\beta$-D-1-thiogalactopyranoside (IPTG) for 2, 3, 4, 6 and $8 \mathrm{~h}$, respectively.

expressed in a prokaryotic expression system, we cloned trCOX-2 and constructed a prokaryotic expression plasmid. As shown in Fig. 3, the 771 bp PCR product encoding the C-terminal segment of human COX-2 (including 257 amino acid residents) was cloned successfully and inserted into the prokaryotic expression vector pET28b(+). Positive recombinant plasmids were confirmed with digestion using BamHI and HindIII enzymes (Fig. 3). The sequencing results provided further evidence of successful construction of the recombinant pET28b-trCOX-2 expression plasmid and confirmed the presence of two 6xHis-tags, located at both the $\mathrm{N}$ - and C-terminus

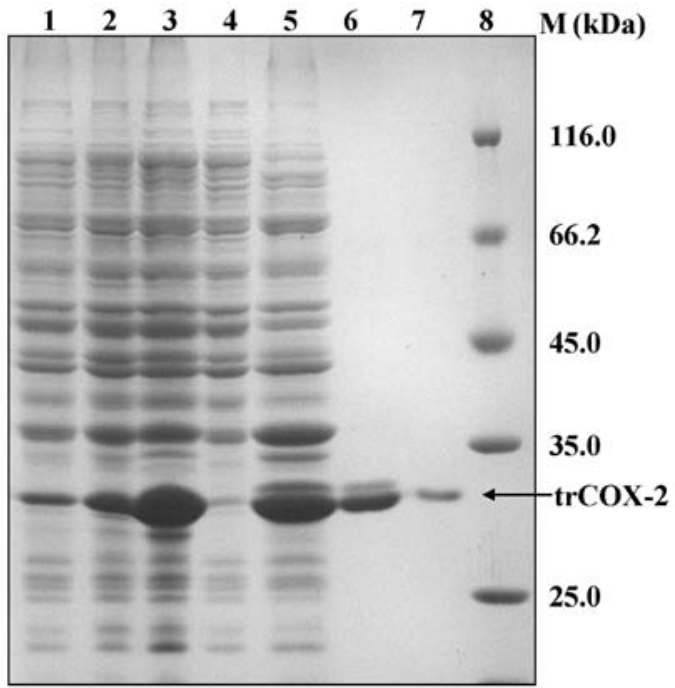

Figure 5. Analysis of purification and renaturation of truncated human cyclooxygenase-2 (trCOX-2) by $12 \%$ SDS-PAGE. Lane 1, cell lysate of pET28b-trCOX-2/ BL21(DE3) without induction; lane 2, total cell lysate of pET28b-trCOX-2/ BL21(DE3) induced by isopropyl $\beta$-D-1-thiogalactopyranoside (IPTG) for $4 \mathrm{~h}$; lane 3, precipitate from the cell lysate of pET28b-trCOX-2/BL21(DE3) induced by IPTG for $4 \mathrm{~h}$; lane 4 , supernatant of the cell lysate of pET28btrCOX-2/BL21(DE3) induced by IPTG for $4 \mathrm{~h}$; lane 5 , the soluble denatured inclusion body proteins; lane 6 , purified trCOX-2 from denatured samples; lane 7, renatured trCOX-2; and lane 8, protein molecular weight standard.

Table I. Purification of trCOX-2 from E. coli BL21(DE3).

\begin{tabular}{lcc}
\hline Steps & $\begin{array}{c}\text { Total products } \\
(\mathrm{mg} / \mathrm{l})^{\mathrm{a}}\end{array}$ & $\begin{array}{c}\text { Yield rate } \\
(\%)\end{array}$ \\
\hline Crude inclusion bodies & 800 & 100 \\
After $\mathrm{Ni}^{2+}$-NTA purification & 75 & 9.4 \\
Renaturation protein & 35 & 4.4 \\
\hline
\end{tabular}

${ }^{\mathrm{a}} \mathrm{mg} / \mathrm{l}$ stands for the total product weight from 1 liter culture medium. trCOX-2, truncated human cyclooxygenase-2; E. coli, Escherichia coli.

of trCOX-2. The full-length of the fusion protein with His-tags, trCOX-2, was 305 amino acids (34.4 kDa).

Expression and purification of trCOX-2. To obtain human trCOX-2 protein, competent E. coli BL21(DE3) cells were transformed with pET28b-trCOX-2 to prepare $E$. coli trCOX-2/BL21(DE3) that could express human trCOX-2. We found that the expression level of the trCOX-2 protein was very high after IPTG induction, as detected by SDS-PAGE (Fig. 4). In addition, the expression of target proteins reached the highest level (up to $31 \%$ of the total $E$. coli protein) at $4 \mathrm{~h}$ after IPTG induction (Fig. 4), but they were expressed as inclusion bodies as they were found in the pellets of cell lysates (Fig. 5). In order to purify trCOX-2, the pellets containing the inclusion bodies were first washed with Triton X-100 and $2 \mathrm{M}$ urea to obtain crude inclusion bodies, which were then solubilized using ureadenaturation. The soluble inclusion body proteins with His-tags were then subjected to affinity purification. SDS-PAGE analysis of the eluted fractions revealed that a single band of approximately $34 \mathrm{kDa}$ was detected (Fig. 5). The purity of the products 
$\mathbf{A}$

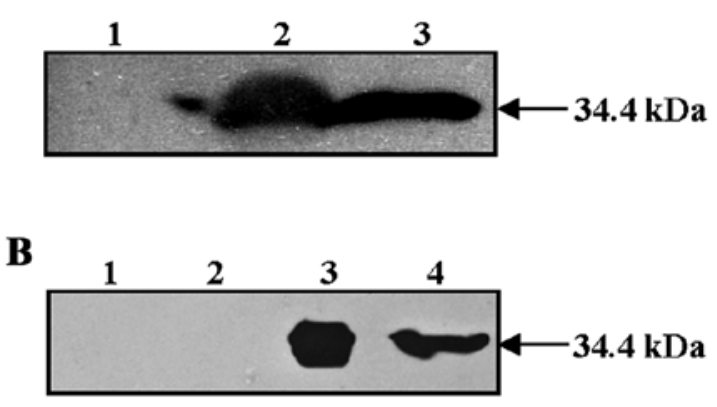

C

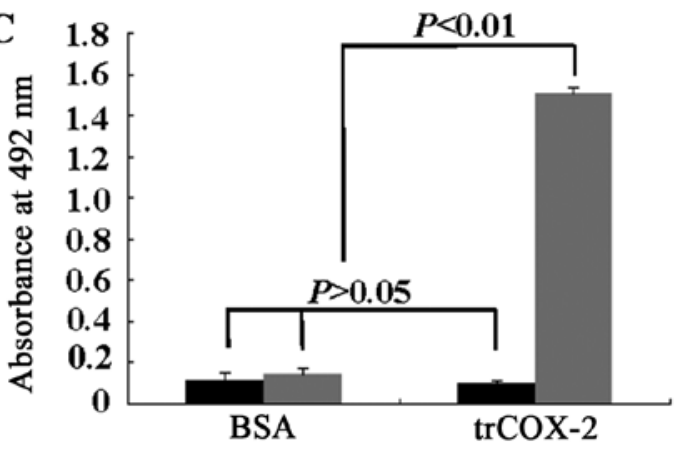

Anti-COX-1 antibody $\square$ Anti-COX-2 antibody

Figure 6. Identification of recombinant truncated human cyclooxygenase-2 (trCOX-2) protein by western blot and ELISA assays. (A) Western blot analysis of trCOX-2 with anti-His-tag antibody. Samples were loaded as follows: lane 1, pET28b-trCOX-2/BL21(DE3) without induction; lane 2, pET28b-trCOX-2/BL21(DE3) induced by isopropyl $\beta$-D-1-thiogalactopyranoside (IPTG) for $4 \mathrm{~h}$; and lane 3, recombinant trCOX-2 protein. (B) Western blot analysis of trCOX-2 with anti-COX-2 antibody. Samples were loaded as follows: lane 1, BL21(DE3); lane 2, pET28b-trCOX-2/BL21(DE3) without induction; lane 3, pET28b-trCOX-2/BL21(DE3) induced by IPTG for $4 \mathrm{~h}$; and lane 4, recombinant trCOX-2 protein. (C) ELISA assay of trCOX-2 with anti-COX-1 and COX-2 antibodies.

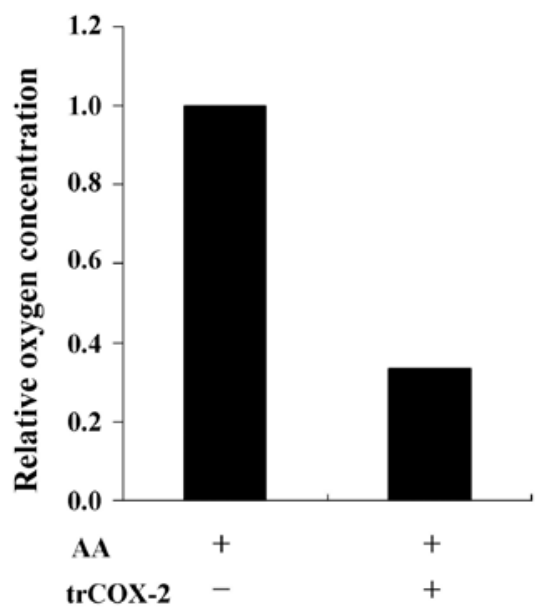

Figure 7. Cyclooxygenase (COX) assay of truncated human COX-2 (trCOX-2). COX activity measured by relative oxygen concentration. A lower final oxygen concentration indicates greater oxygen consumption and higher COX activity.

was $>95 \%$, as estimated by gel analysis using Bandscan software. The eluted fractions containing the protein of interest were refolded by dilution in refolding buffer to prepare purified active trCOX-2. The purification of human trCOX-2 is summarized in Table I. This process yielded approximately $35 \mathrm{mg}$ of purified protein from 1 liter of E. coli culture.

Identification of purified trCOX-2. Western blot analyses using anti-His-tag antibody (Fig. 6A) and anti-COX-2 antibody (Fig. 6B) were used to further identify recombinant trCOX-2, because recombinant trCOX-2 contains His-tags at both the $\mathrm{N}$ - and $\mathrm{C}$-terminus. As shown in Fig. 6, both the cell lysate of $E$. coli pET28b-trCOX-2/BL21(DE3) induced by IPTG for $4 \mathrm{~h}$ and the trCOX-2 protein showed a clear single band at approximately $34 \mathrm{kDa}$ after incubation with anti-His-tag antibody (Fig. 6A) and anti-COX-2 antibody (Fig. 6B), respectively. Protein bands were not found in samples without IPTG induction. These results suggested trCOX-2 with His-tags was expressed successfully in $E$. coli pET28b-trCOX-2/BL21(DE3) cells induced by IPTG for $4 \mathrm{~h}$ and that purified trCOX-2 was obtained.
In order to examine the antigenicity and binding activity of prepared trCOX-2 to anti-COX-2 or anti-COX-1 antibody, an ELISA assay was performed. As shown in Fig. 6C, the ELISA assay results demonstrated that the recombinant trCOX-2 showed selective binding to anti-COX-2 antibody. The absorbance at $490 \mathrm{~nm}$ due to binding between recombinant trCOX-2 and anti-COX-2 antibody was significantly higher $(\mathrm{P}<0.01)$ than the absorbance observed for each of three controls: the binding of BSA, an irrelevant antigen, to anti-COX-2 antibody; BSA binding to anti-COX-1 antibody; recombinant trCOX-2 binding to anti-COX-1 antibody. Moreover, only low absorbance readings were obtained for anti-COX-1 antibody binding to either BSA or trCOX-2 and these results were not significantly different from the results for BSA binding to anti-COX-2 antibody $(\mathrm{P}>0.05)$. These results suggest that purified recombinant trCOX-2 retains the same antigenicity as human COX-2 and has selective binding affinity to anti-COX-2 antibody.

Purified trCOX-2 exhibits $C O X$ activity. To verify the catalytic activity of trCOX-2, COX activity was conducted by recording oxygen consumption. The oxygenation and cyclization of AA within the $\mathrm{COX}$ active site during formation of $\mathrm{PGG}_{2}$ consumes oxygen in the reaction whereby $\mathrm{COX}-2$ catalyzes the conversion of AA to PGs. The oxygen consumption of COX activity can be monitored using a detector to measure dissolved oxygen and recorded as the relative variation in oxygen concentration. As shown in Fig. 7, compared with the control group without trCOX-2 protein, the final oxygen concentration was apparently lower in the test group after addition of trCOX-2 protein. This result demonstrates that the purified trCOX-2 still exhibited COX activity, further confirming our speculation that trCOX-2 possessed COX activity.

\section{Discussion}

In this study, we report a strategy with which to express trCOX-2 in E. coli BL21(DE3) cells. For the purpose of achieving high-level expression in $E$. coli cells, human COX-2 was truncated to remove the stretch of residues from the N-terminus, with the aim to reduce the size and structural complexity of 
human COX-2, while maintaining enzyme activity. Homology modeling and molecular docking results predicted that trCOX-2 retained the active site in its 3D structure and that AA could still bind to the hydrophobic groove, as shown in Figs. 1 and 2. The fusion trCOX-2 protein with His-tags was efficiently expressed and was localized to inclusion bodies after IPTG induction. Through denaturation, purification and renaturation, we successfully obtained soluble trCOX-2 proteins that were recognized specifically by anti-COX-2 antibody but not by antiCOX-1 antibody. Moreover, the COX assays indicated that the trCOX-2 maintained COX activity. This human COX-2 preparation strategy provides a reliable method to obtain functional products and is a valuable guide for prokaryotic expression of eukaryotic membrane protein.

COX-2 is a rate-limiting key enzyme which catalyzes the conversion of AA into PGs. The expression of COX-2 is intimately involved in a number of pathologies, such as inflammation, pain and various epithelial tumors $(39,40)$. In addition, COX-2 closely correlates with and is widely involved in most processes giving rise to malignant tumor development, including the formation of carcinogens, tumor promotion, inhibition of apoptosis, stimulation of angiogenesis, invasion, metastasis and drug-resistance (11-13). COX-2 overexpression has been regarded as an early event in carcinogenesis (10-12). Therefore, COX-2 is an important target for anti-inflammation and anticancer therapies. To develop these therapies, an effective and inexpensive expression strategy to obtain bioactive and functional human COX-2 would be a key step.

Although different types of recombinant proteins have been successfully isolated in various expression systems, including $E$. coli cells $(14,15)$, previous studies have shown that functional COX-2 has been most often expressed in insect/ baculovirus expression systems for structure determination and function analysis in vitro (16-19). However, several advantages of prokaryotic systems over insect/baculovirus expression systems favor use of a prokaryotic system for high yield production of COX-2. E. coli is one of the most widely used expression hosts, coupled with the fact that techniques for protein overexpression in E. coli are well developed. Because protein synthesis rates are generally much faster in prokaryotes than in eukaryotes (20), for large-scale production of proteins, bacterial expression hosts such as $E$. coli are preferred due to its rapid growth rate, capacity for continuous fermentation, high-level expression of target protein after induction and relatively low cost (14,20-23). In this study, E. coli BL21(DE3) and $\mathrm{pET} 28 \mathrm{~b}(+)$ were used to achieve overexpression of functional truncated human COX-2. We obtained approximately $350 \mathrm{mg}$ of renatured trCOX-2 from 10 liters of culture using this prokaryotic expression system (Table I). Previous studies have shown that 10 liters of fermentation cultures of insect cells only yielded $35 \mathrm{mg}$ of COX-2 (17), showing that COX-2 was extracted almost 10-fold more efficiently in our prokaryotic expression system than using an insect/baculovirus expression system. Therefore, the expression system described in this study guarantees a high yield of human COX-2 protein.

The smaller size and simpler protein structure of human recombinant $\mathrm{COX}-2$ protein has permitted its effective expression in prokaryotic expression systems $(20-23,36,37)$. Information from the crystal structure of COX-2 has revealed that key active residues (Tyr-385, Phe-381, Val-523, Glu-524 and Ser-530) are found in the catalytic domain within the C-terminus. In order to obtain high-level expression of human COX-2 in E. coli cells, the truncated form lacking the $\mathrm{N}$-terminus containing 257 residues of the $\mathrm{C}$-terminus was prepared to maximally reduce the size and structural complexity of human COX-2 while maintaining its enzyme activity. As a result, trCOX-2 protein with $\mathrm{COX}$ activity was expressed successfully at a high-level in E. coli cells.

In our E. coli expression system, the eukaryotic membrane proteins are inclined to be expressed in insoluble forms known as inclusion bodies (20). Inclusion bodies are aggregations of proteins which are largely protected from proteolytic degradation by host cell enzymes $(14,20)$. Through proper denaturant and efficient renaturant methods, high-purity target proteins may be retrieved in large amounts (20-26). The key step to obtaining a large quantity of functional protein is the establishment of an economical and highly effective method to dissolve and renature the inclusion bodies (24-26). For the first time, to the best of our knowledge, we obtained functional trCOX-2 using this prokaryotic expression system through denaturation and renaturation with buffer D and E, respectively (see Materials and methods).

In conclusion, our study describes a prokaryotic functional expression strategy to generate high yields of truncated and enzymatically active human COX-2. The trCOX-2 product is useful for designing COX-2 inhibitors and anti-COX-2 antibodies. Furthermore, this method provides a practical foundation to achieve overexpression of eukaryotic membrane proteins in an E. coli expression system.

\section{Acknowledgements}

This study was partly supported by the National Natural Science Foundation of China (nos. 31170882, 31570934, 81428006), the S\&T Development Planning Program of Jilin Province (nos. 20111806, 20150414027GH, 20160101213JC) and the Fundamental Research Funds for the Central Universities (no. 451160306023).

\section{References}

1. Chandrasekharan NV and Simmons DL: The cyclooxygenases. Genome Biol 5: 241, 2004.

2. Rowlinson SW, Crews BC, Lanzo CA and Marnett LJ: The binding of arachidonic acid in the cyclooxygenase active site of mouse prostaglandin endoperoxide synthase-2 (COX-2). A putative $\mathrm{L}$-shaped binding conformation utilizing the top channel region. J Biol Chem 274: 23305-23310, 1999.

3. Vecchio AJ and Malkowski MG: The structural basis of endocannabinoid oxygenation by cyclooxygenase-2. J Biol Chem 286: 20736-20745, 2011.

4. Vecchio AJ, Orlando BJ, Nandagiri R and Malkowski MG: Investigating substrate promiscuity in cyclooxygenase-2: the role of Arg-120 and residues lining the hydrophobic groove. J Biol Chem 287: 24619-24630, 2012

5. Dong L, Vecchio AJ, Sharma NP, Jurban BJ, Malkowski MG and Smith WL: Human cyclooxygenase-2 is a sequence homodimer that functions as a conformational heterodimer. J Biol Chem 286: 19035-19046, 2011.

6. Vecchio AJ, Simmons DM and Malkowski MG: Structural basis of fatty acid substrate binding to cyclooxygenase-2. J Biol Chem 285: 22152-22163, 2010.

7. Kudalkar SN, Nikas SP, Kingsley PJ, Xu S, Galligan JJ, Rouzer CA, Banerjee S, Ji L, Eno MR, Makriyannis A, et al: 13-Methylarachidonic acid is a positive allosteric modulator of endocannabinoid oxygenation by cyclooxygenase. J Biol Chem 290: 7897-7909, 2015. 
8. Tanabe $\mathrm{T}$ and Tohnai $\mathrm{N}$ : Cyclooxygenase isozymes and their gene structures and expression. Prostaglandins Other Lipid Mediat 68-69: 95-114, 2002.

9. Li G, Han C, Xu L, Lim K, Isse K and Wu T: Cyclooxygenase-2 prevents Fas-induced liver injury through up-regulation of epidermal growth factor receptor. Hepatology 50: 834-843, 2009.

10. Ghosh N, Chaki R, Mandal V and Mandal SC: COX-2 as a target for cancer chemotherapy. Pharmacol Rep 62: 233-244, 2010.

11. Khan Z, Khan N, Tiwari RP, Sah NK, Prasad GB and Bisen PS: Biology of Cox-2: an application in cancer therapeutics. Curr Drug Targets 12: 1082-1093, 2011.

12. Misra $\mathrm{S}$ and Sharma K: COX-2 signaling and cancer: new players in old arena. Curr Drug Targets 15: 347-359, 2014.

13. Kurtova AV, Xiao J, Mo Q, Pazhanisamy S, Krasnow R, Lerner SP, Chen F, Roh TT, Lay E, Ho PL, et al: Blocking $\mathrm{PGE}_{2}$-induced tumour repopulation abrogates bladder cancer chemoresistance. Nature 517: 209-213, 2015.

14. Yin J, Li G, Ren X and Herrler G: Select what you need: a comparative evaluation of the advantages and limitations of frequently used expression systems for foreign genes. J Biotechnol 127: 335-347, 2007.

15. Wu Y, Zou D, Cao Y, Yao N, Wang J, Wang W, Jiang H and Li G: Expression and purification of a human anti-cyclin D1 single-chain variable fragment antibody AD5 and its characterization. Int J Mol Med 32: 1451-1457, 2013.

16. Percival MD, Bastien L, Griffin PR, Kargman S, Ouellet M and O'Neill GP: Investigation of human cyclooxygenase-2 glycosylation heterogeneity and protein expression in insect and mammalian cell expression systems. Protein Expr Purif 9: 388-398, 1997.

17. Gierse JK: Purification of recombinant human COX-1 and COX-2. Methods Mol Biol 644: 21-29, 2010.

18. Gierse JK, McDonald JJ, Hauser SD, Rangwala SH, Koboldt CM and Seibert K: A single amino acid difference between cyclooxygenase-1 (COX-1) and -2 (COX-2) reverses the selectivity of COX-2 specific inhibitors. J Biol Chem 271: 15810-15814, 1996.

19. Cromlish WA, Payette P, Culp SA, Ouellet M, Percival MD and Kennedy BP: High-level expression of active human cyclooxygenase-2 in insect cells. Arch Biochem Biophys 314: 193-199, 1994.

20. Gul N, Linares DM, Ho FY and Poolman B: Evolved Escherichia coli strains for amplified, functional expression of membrane proteins. J Mol Biol 426: 136-149, 2014.

21. Liu B, Li G, Sui X, Yin J, Wang H and Ren X: Expression and functional analysis of porcine aminopeptidase $\mathrm{N}$ produced in prokaryotic expression system. J Biotechnol 141: 91-96, 2009.

22. Arbabi-Ghahroudi M, Tanha J and MacKenzie R: Prokaryotic expression of antibodies. Cancer Metastasis Rev 24: 501-519, 2005.

23. Sheibani N: Prokaryotic gene fusion expression systems and their use in structural and functional studies of proteins. Prep Biochem Biotechnol 29: 77-90, 1999.

24. Kim CS and Lee EK: Effects of operating parameters in in vitro renaturation of a fusion protein of human growth hormone and glutathione $\mathrm{S}$ transferase from inclusion body. Process Biochem 36: 111-117, 2000.

25. Li GY, Zou DS, Zhou LH and Cao YH: Expression and purification of recombinant human cyclin D1 in E. coli BL21. J Jilin Univ 44: 839-843, 2006.
26. Cao YH, Xu JJ, Chen Y, Wang Q, Feng J, Hao DY and Li GY: Prokaryotic expression, purification and renaturation of recombinant human CDK4. J Jilin Univ 46: 992-996, 2008.

27. Xiang Y, Wang HY, Lei W and Sun M: The expression and purification of COX-2 in Escherichia coli. J Southwest Univ 12: $121-125,2008$

28. Blobaum AL, Xu S, Rowlinson SW, Duggan KC, Banerjee S, Kudalkar SN, Birmingham WR, Ghebreselasie K and Marnett LJ: Action at a distance: mutations of peripheral residues transform rapid reversible inhibitors to slow, tight binders of cyclooxygenase-2. J Biol Chem 290: 12793-12803, 2015.

29. Arnold K, Bordoli L, Kopp J and Schwede T: The SWISS-MODEL workspace: a web-based environment for protein structure homology modelling. Bioinformatics 22: 195-201, 2006

30. Guex N, Peitsch MC and Schwede T: Automated comparative protein structure modeling with SWISS-MODEL and Swiss-PdbViewer: a historical perspective. Electrophoresis 30 (Suppl 1): S162-S173, 2009.

31. Kiefer F, Arnold K, Künzli M, Bordoli L and Schwede T: The SWISS-MODEL repository and associated resources. Nucleic Acids Res 37 (Database): D387-D392, 2009.

32. Biasini M, Bienert S, Waterhouse A, Arnold K, Studer G, Schmidt T, Kiefer F, Gallo Cassarino T, Bertoni M, Bordoli L, et al: SWISS-MODEL: modelling protein tertiary and quaternary structure using evolutionary information. Nucleic Acids Res 42: W252-W258, 2014.

33. Grosdidier A, Zoete V and Michielin O: Fast docking using the CHARMM force field with EADock DSS. J Comput Chem 32: 2149-2159, 2011.

34. Grosdidier A, Zoete V and Michielin O: SwissDock, a protein-small molecule docking web service based on EADock DSS. Nucleic Acids Res 39: W270-W277, 2011.

35. Nandana V, Singh S, Singh AN and Dubey VK: Procerain B, a cysteine protease from Calotropis procera, requires N-terminus pro-region for activity: cDNA cloning and expression with pro-sequence. Protein Expr Purif 103: 16-22, 2014.

36. Doray B, Chen CD and Kemper B: N-terminal deletions and His-tag fusions dramatically affect expression of cytochrome p450 2C2 in bacteria. Arch Biochem Biophys 393: 143-153, 2001

37. Housaindokhta MR, Bozorgmehr MR, Hosseini HE, Jalal R, Asoodeh A, Saberi M, Haratipour Z and Monhemi H: Structural properties of the truncated and wild types of Taka-amylase: a molecular dynamics simulation and docking study. J Mol Catal B Enzym 95: 36-40, 2013.

38. Wilkins MR, Gasteiger E, Bairoch A, Sanchez JC, Williams KL, Appel RD and Hochstrasser DF: Protein identification and analysis tools in the ExPASy server. Methods Mol Biol 112: 531-552, 1999.

39. Kim SF, Huri DA and Snyder SH: Inducible nitric oxide synthase binds, S-nitrosylates, and activates cyclooxygenase-2. Science 310: 1966-1970, 2005.

40. Gierse JK, Hauser SD, Creely DP, Koboldt C, Rangwala SH, Isakson PC and Seibert K: Expression and selective inhibition of the constitutive and inducible forms of human cyclo-oxygenase. Biochem J 305: 479-484, 1995. 